Discrete Math. Appl., Vol. 7, No. 3, pp. 223-229 (1997)

(C) VSP 1997.

\title{
Fibre products of hyperelliptic curves and geometric Goppa codes ${ }^{*}$
}

\author{
S. A. STEPANOV and F. ÖZBUDAK
}

Abstract - The purpose of this paper is to extend the results of the first author on construction of fairly long geometric Goppa codes over $F_{q}\left(q=p^{v}\right.$ and $v>1$ is even) with rather good parameters to the case of finite fields $F_{q}$ consisting of $q=p^{v}$ elements, where $v>1$ is an odd integer.

The work was supported by Bilkent University, Ankara, Turkey.

The first author was partially supported by the Russian Foundation for Basic Research, grant 94-0101206-a.

\section{INTRODUCTION}

Recall the basic ideas of the Goppa construction (see $[2,3]$ ) of the linear $[n, k, d]_{q}$-codes associated with a smooth projective curve $X$ of genus $g=g(X)$ defined over a finite field $F_{q}$. Let $\mathcal{P}=\left\{P_{1}, \ldots, P_{n}\right\}$ be a set of $F_{q}$-rational points of $X$ and

$$
D_{0}=P_{1}+\ldots+P_{n} .
$$

Let $D$ be a $F_{q}$-rational divisor on $X$ whose support is disjoint with $D_{0}$. We consider the vector $F_{q}$-space of rational functions on $X$

$$
L(D)=\left\{f \in F_{q}(X)^{*} \mid(f)+D \geq 0\right\} \cup\{0\},
$$

and denote its dimension over $F_{q}$ by $l(D)$. The linear $[n, k, d]$-code $C=C\left(D_{0}, D\right)$ associated with the pair $\left(D_{0}, D\right)$ is the image of the linear evaluation map

$$
\mathrm{Ev}: L(D) \rightarrow F_{q}^{n}, \quad f \mapsto\left(f\left(x_{1}\right), \ldots, f\left(x_{n}\right)\right) .
$$

Such a $q$-ary linear code is called a geometric Goppa code. If $\operatorname{deg} D<n$, then the map Ev is an embedding, hence $k=\operatorname{dim} C=l(D)$ and by the Riemann-Roch theorem

$$
k \geq \operatorname{deg} D-g+1
$$

in particular, if $2 g-2<\operatorname{deg} D<n$, then

$$
k=\operatorname{deg} D-g+1 \text {. }
$$

Moreover, we have

$$
d \geq n-\operatorname{deg} D .
$$

${ }^{*}$ UDC 519.72. Originally published in Diskretnaya Matematika (1997) 9, No. 3 (in Russian). Received August 13, 1996. Translated by the authors. 
Theorem 1. Let $v>1$ be an odd number, $F_{q}$ be a finite field of characteristic $p>2$ consisting of $q=p^{v}$ elements, and let $s$ be an integer such that

$$
1 \leq s<\frac{2 p^{v}+4}{p^{(v-1) / 2}(p+1)-2} .
$$

Moreover, let $r$ be an integer such that

$$
2^{s-2}\left(\left(p^{(v-1) / 2}(p+1)-2\right) s-4\right)<r<2^{s} p^{v} .
$$

Then there exists a linear $[n, k, d]_{q}$-code with parameters

$$
\begin{aligned}
& r<n \leq 2^{s} p^{v} \\
& k=r-2^{s-2}\left(\left(p^{(v-1) / 2}(p+1)-2\right) s-4\right), \\
& d \geq n-r .
\end{aligned}
$$

Corollary 1. Under the conditions of Theorem 1 , there exists a linear $[n, k, d]_{q}$-code with the relative parameters $R=k / n$ and $\delta=d / n$ such that

$$
R \geq 1-\delta-\frac{2^{s-2}\left(\left(p^{(v-1) / 2}(p+1)-2\right) s-4\right)}{n} .
$$

In particular, for $n=2^{s} p^{v}$ we have

$$
R \geq 1-\delta-\frac{\left(p^{(v-1) / 2}(p+1)-2\right) s-4}{4 p^{v}} .
$$

\section{NOTATION AND LEMMAS}

Let $\bar{F}_{q}$ be an algebraic closure of the field $F_{q}$ and $A^{s+1}$ be the $(s+1)$-dimensional affine space over $\bar{F}_{q}$.

Lemma 1. Let $f_{1}, f_{2}, \ldots, f_{s} \in F_{q}[x]$ be pairwise coprime square-free monic polynomials of the same degree $m \geq 3$ and $Y$ be the fibre product in $A^{s+1}$ given over $F_{q}[x]$ defined by the equations

$$
\begin{gathered}
z_{1}^{2}=f_{1}(x), \\
z_{2}^{2}=f_{2}(x), \\
\cdots \\
z_{s}^{2}=f_{s}(x) .
\end{gathered}
$$

Then the genus $g=g(X)$ of the smooth projective model $X$ of the curve $Y$ is

$$
g= \begin{cases}(m s-3) 2^{s-2}+1 & \text { if } m \text { is odd } \\ (m s-4) 2^{s-2}+1 & \text { if } m \text { is even }\end{cases}
$$


Proof. Let $I$ be the ideal of the curve $Y$ in $\bar{F}_{q}\left[x, z_{1} \ldots, z_{s}\right]$ and $\bar{Y}$ be the projective closure of $Y$ in $P^{s+1}$. The homogeneous ideal of $\bar{Y}$ in $\bar{F}_{q}\left[x_{0}, x, z_{1}, \ldots, z_{s}\right]$ has the form $I_{h}=\left\{f_{h} \mid f \in I\right\}$, where $f_{h}$ is the homogenization of $f$, i.e.,

$$
f_{h}\left(x_{0}, x, z_{1}, \ldots, z_{s}\right)=f\left(x / x_{0}, z_{1} / x_{0}, \ldots, z_{s} / x_{0}\right) x_{0}^{\operatorname{deg} f} .
$$

Thus, $\bar{Y}=Y \cup\{(0,0, \pm 1, \pm 1, \ldots, \pm 1)\}$ as a set, and the curve $\bar{Y}$ is singular at the $2^{s-1}$ points $P_{i} \in\{(0,0,1, \pm 1, \ldots, \pm 1)\}$ in general.

Let $X$ be a normalization of $\bar{Y}$ which in the same time is a non-singular model of $\bar{Y}$ (see, for example [3], Chapter 2, 5.3). There exists a finite morphism (regular map) $\varphi_{1}: X \rightarrow \bar{Y}$ and a composition of $\varphi_{1}$ with $\varphi_{2}$, where $\varphi_{2}: \bar{Y} \rightarrow P^{1}$ via $\left(x_{0}, x, z_{1}, \ldots, z_{k}\right) \mapsto\left(x_{0}, x\right)$ gives a morphism $\varphi: X \rightarrow P^{1}$ of degree $2^{s}$ (see, for example [3], Chapter 2, 3.1). Since $\bar{Y}$ has $2^{s-1}$ points $P_{i}, 1 \leq i \leq 2^{s-1}$, at the hypersurface $x_{0}=0$, the set $\varphi^{-1}(0,1)$ consists of $2^{s}$ or $2^{s-1}$ points $\left\{Q_{i}\right\} \subseteq X$.

Let $\Omega[\bar{Y}]$ be the space of regular differential forms on $\bar{Y}$. The space $\Omega[\bar{Y}]$, considered as a $\bar{F}_{q}\left[x, z_{1}, \ldots, z_{s}\right]$-module, is generated by $d x$ and $d z_{i}, 1 \leq i \leq s$. Since $z_{i}^{2}=f_{i}(x)$, the space $\Omega[\bar{Y}]$, considered as a $\bar{F}_{q}[x]$-module, is generated by $d x$ and $d x /\left(z_{i_{1}} \ldots z_{i_{\sigma}}\right)$, where $1 \leq i_{1}<\ldots<i_{\sigma} \leq s$. Next, since $\varphi_{1}$ is a morphism, the space $\Omega[X]$ is a submodule of $\Omega[\bar{Y}]$, hence any differential form $\omega \in \Omega[X]$ has one of the form

$$
\omega=F(x) d x, \quad \omega=\frac{F_{i_{1} \ldots, i_{\sigma}}(x) d x}{z_{i_{1}} \ldots z_{i_{\sigma}}}
$$

with $F, F_{i_{1}, \ldots, i_{\sigma}} \in \bar{F}_{q}[x]$. Thus, any regular differential form in $\Omega[\bar{Y}]$ is regular at any point of $X$, possibly except $Q_{i} \in \varphi^{-1}(0,1)$.

Let $x$ be the coordinate on $P^{1}$, then $u=x^{-1}$ is a local parameter at the point $(0,1)$ at infinity. Since $x$ is a rational function on $P^{1}$, it defines the divisor $(x) \in \operatorname{Div}\left(P^{1}\right)$. Denoting $\varphi^{-1}(x) \in \bar{F}_{q}(X)$ by $x$ and its divisor by $(x)$ again, we get the pull-back divisor $(x) \in \operatorname{Div}(X)$.

Since $\varphi^{-1}(0,1)$ consists of $2^{s}$ or $2^{s-1}$ points $Q_{i}$, we have $v_{Q_{i}}(u)=1$ or $v_{Q_{i}}(u)=2$, therefore $v_{Q_{i}}(x)=-1$ or $v_{Q_{i}}(x)=-2$. If $F(x)$ is a regular function on $X$, we have $v_{Q_{i}}(F(x) d x)=-(\operatorname{deg} F(x)+2)$ or $-(2 \operatorname{deg} F(x)+3)$ respectively. Thus, $F(x) d x \notin \Omega[X]$ for any $F(x) \in \bar{F}_{q}[X]$.

If $m$ is even, then there are two cases:

(1) $v_{Q_{i}}(x)=-1$ and $v_{Q_{i}}\left(z_{j}\right)=-m / 2$ for any $j=1, \ldots, s$,

(2) $v_{Q_{i}}(x)=-2$ and $v_{Q_{i}}\left(z_{j}\right)=-m$ for any $j=1, \ldots, s$.

Since

$$
v_{Q_{i}}\left(\frac{F_{i_{1}, \ldots, i_{\sigma}}(x) d x}{z_{i_{1}} \ldots z_{i_{\sigma}}}\right)=v_{Q_{i}}(x) \operatorname{deg} F_{i_{1}, \ldots, i_{\sigma}}(x)+\left(v_{Q_{i}}(x)-1\right)-\sigma v_{Q_{i}}\left(z_{j}\right)
$$

for any $j=1, \ldots, s$, we have

$$
v_{Q_{i}}\left(\frac{F_{i_{1}, \ldots, i_{\sigma}}(x) d x}{z_{i_{1}} \ldots z_{i_{\sigma}}}\right)=\frac{m \sigma}{2}-\operatorname{deg} F_{i_{1}, \ldots, i_{\sigma}}(x)-2,
$$


or

$$
v_{Q_{i}}\left(\frac{F_{i_{1}, \ldots, i_{\sigma}}(x) d x}{z_{i_{1}} \ldots z_{i_{\sigma}}}\right)=m \sigma-2 \operatorname{deg} F_{i_{1}, \ldots, i_{\sigma}}(x)-3
$$

respectively. Thus,

$$
\frac{F_{i_{1}, \ldots, i_{\sigma}}(x) d x}{z_{i_{1}} \ldots z_{i_{\sigma}}} \in \Omega[X]
$$

if and only if

$$
\operatorname{deg} F_{i_{1}, \ldots, i_{\sigma}}(x) \leq \frac{m \sigma}{2}-2
$$

or

$$
\operatorname{deg} F_{i_{1}, \ldots, i_{\sigma}}(x) \leq \frac{m \sigma}{2}-\frac{3}{2}
$$

respectively. Since $m$ is even the second inequality is equivalent to the first one.

If $m$ is odd and $v_{Q_{i}}(x)=-1$, then $v_{Q_{i}}\left(z_{j}^{2}\right)=2 v_{Q_{i}}\left(z_{j}\right)=-m$ and we arrive at a contradiction. Thus, only one case is possible, where $v_{Q_{i}}(x)=-2$. In this case,

$$
\frac{F_{i_{1}, \ldots, i_{\sigma}}(x) d x}{z_{i_{1}} \ldots z_{i_{\sigma}}} \in \Omega[X]
$$

if only

$$
\operatorname{deg} F_{i_{1}, \ldots, i_{\sigma}}(x) \leq \begin{cases}(m \sigma-4) / 2 & \text { if } \sigma \text { is even, } \\ (m \sigma-3) / 2 & \text { if } \sigma \text { is odd. }\end{cases}
$$

Since $X$ is non-singular, we have

$$
g=\operatorname{dim}_{F_{q}} \Omega[X]
$$

Thus, if $m$ is even, then

$$
g=\frac{1}{2} \sum_{\sigma=1}^{s} \sum_{1 \leq i_{1}<i_{2}, \ldots<i_{\sigma} \leq s}(m \sigma-2)=(m s-4) 2^{s-2}+1,
$$

and if $m$ is odd, then

$$
\begin{aligned}
g & =\frac{1}{2} \sum_{\sigma=1}^{s} \sum_{1 \leq i_{1}<i_{2}, \ldots<i_{\sigma} \leq s}(m \sigma-2)+\frac{1}{2} \sum_{\sigma=1}^{s} \sum_{1 \leq i_{1}<i_{2}, \ldots<i_{\sigma} \leq s s}(m \sigma-1) \\
& =(m s-3) 2^{s-2}+1,
\end{aligned}
$$

where the summation is taken over odd $\sigma$ only.

This completes the proof.

Lemma 2. Let $v>1$ be an odd number, $F_{q}$ be a finite field of characteristic $p>2$ with $q=p^{v}$ elements, and let $f \in F_{q}[x]$ be the polynomial

$$
f(x)=\left(x+x^{p^{(v-1) / 2}}\right)\left(x+x^{p^{(v+1) / 2}}\right) .
$$

If $c$ is a non-zero element of $F_{q}$, then the polynomials $f(x)$ and $f(x+c)$ are relatively prime. 
Proof. Let $\mu=(v-1) / 2$ and $f^{\prime}(x)=x^{\prime{ }^{\prime \prime}}+x, f^{\prime \prime}(x)=x^{\mu^{\mu+1}}+x$ so that $f^{\prime}(x) f^{\prime \prime}(x)=f(x)$. We shall prove that $\left(f^{\prime}(x), f^{\prime}(x+c)\right)=(1)$ and $\left(f^{\prime}(x), f^{\prime \prime}(x+c)\right)=(1)$ for any $c \in F_{p^{*}}^{*}$. This will imply that $(f(x), f(x+c))=(1)$ for any $c \in F_{p^{*}}^{*}$.

Observe that the principal ideal $I^{\prime}$ generated by $f^{\prime}(x)$ and $f^{\prime}(x+c)$ is equal to

$$
I^{\prime}=\left(x^{p^{\mu}}+x, x^{p^{\mu}}+x+c^{\mu^{\mu}}+c\right)
$$

The equation

$$
\alpha^{p^{\mu}}+\alpha=0
$$

has no solution in $F_{p^{v}}^{*}$. Otherwise $\alpha^{p^{\mu}-1}=-1$. Then $\alpha^{p^{2 \mu}-1}=1$, since $p$ is odd and hence $2 \mid\left(p^{\mu}+1\right)$. Thus, $\alpha \in F_{p^{\mathrm{gcd}(2 \mu+1.2 \mu)}}=F_{p}$. This implies that $\alpha^{\mu^{\mu}-1}=1 \neq-1$, and we obtain a contradiction.

Observe (using the Euclidean algorithm) that if $k, l, k \geq l$, are positive integers and $c \in F_{p^{v}}$, then the principal ideal $\left(x^{k}+x+c, x^{l}+x\right)$ in $F_{p^{v}}[x]$ satisfies the relation

$$
\left(x^{k}+x+c, x^{l}+x\right)=\left(x^{l}+x,-x^{k-l+1}+x+c\right) .
$$

Similarly,

$$
\left(-x^{k}+x+c, x^{l}+x\right)=\left(x^{l}+x, x^{k-l+1}+x+c\right) .
$$

Combining these relations, we find that if $k \geq 2 l-1$ and $k, l$ are positive integers, then

$$
\left(x^{k}+x+c, x^{l}+x\right)=\left(x^{l}+x, x^{k-2 l+2}+x+c\right) .
$$

By induction, if $l \mid k$ and $c \in F_{p^{v}}^{*}$, then

$$
\left(x^{k}+x+c, x^{l}+x\right)=\left(x^{l}+x,(-1)^{k l l} x^{k / l}+x+c\right) \text {. }
$$

Applying this relation for $k=p^{\mu+1}$ and $l=p^{\mu}$, we find for the ideal $I^{\prime \prime}=\left(f^{\prime \prime}(x+c), f^{\prime}(x)\right)$ that

$$
I^{\prime \prime}=\left(x^{\prime \prime}+x,-x^{\prime \prime}+x+c^{\prime^{\prime \prime+1}}+c\right) \text {. }
$$

Now we observe that $\left(g^{\prime}(x), g^{\prime \prime}(x)\right) \supset\left(g^{\prime}(x),\left(g^{\prime \prime}(x)\right)^{p}\right)$ for any $h^{\prime}, h^{\prime \prime} \in F_{p^{v}}[x]$. Therefore

$$
I^{\prime \prime} \supset J=\left(x^{p^{\prime \prime}}+x,-x^{\prime^{\mu+1}}+x^{\prime^{\mu}}+\gamma^{p^{\mu+1}}+\gamma\right)
$$

where $\gamma=c^{p^{\mu}}$. We can simplify the generators of $J$ as

$$
\begin{aligned}
J & =\left(x^{p^{\prime \prime}}+x,-x^{p^{\mu+1}}-x+\gamma^{p^{\mu+1}}+\gamma\right) \\
& =\left(x^{p^{\prime \prime}}+x, x^{\mu^{\mu+1}}+x-\gamma^{p^{\mu+1}}-\gamma\right) .
\end{aligned}
$$

Let us show that

$$
c^{\mu+1}+c \neq-\gamma^{\mu^{\mu+1}}-\gamma
$$


Since $\gamma=c^{p^{\mu}}$ and $c^{p^{v}}=c \in F_{p^{v}}^{*}$, we can rewrite the inequality (2) in the form

$$
c^{p^{\mu+1}}+c^{p^{\mu}}+2 c \neq 0
$$

The equation

$$
\beta^{p^{\mu+1}}+\beta^{p^{\mu}}+2 \beta=0
$$

has no solution in $F_{p^{v}}^{*}$. Indeed, raising both sides of (4) to the $p^{\mu}$ th power, we obtain

$$
\beta+\beta^{p^{2 \mu}}+\beta^{p^{\mu}}+\beta^{p^{\mu}}=\left(\beta^{p^{\mu}}+\beta\right)^{p^{\mu}}+\left(\beta^{p^{\mu}}+\beta\right)=0 .
$$

Since (1) has no non-zero solution, the last equation also has no solution in $F_{p^{v}}^{*}$.

Now since $f^{\prime \prime}(x+c)=x^{p^{\mu+1}}+x+c^{p^{\mu+1}}+c \in I^{\prime \prime}, x^{p^{\mu+1}}+x-\gamma^{p^{\mu+1}}-\gamma \in J \subseteq I^{\prime \prime}$, and

$$
c^{\mu+1}+c \neq-\gamma^{\mu^{\mu+1}}-\gamma
$$

we conclude that $I^{\prime \prime}=(1)$.

By symmetry $\left(f^{\prime \prime}(x), f^{\prime \prime}(x+c)\right)=(1)$, and $\left(f^{\prime \prime}(x), f^{\prime}(x+c)\right)=(1)$. Using the uniqueness of factorization in $F_{p^{v}}[x]$, we find that

$$
\left(f^{\prime}(x), f^{\prime}(x+c) f^{\prime \prime}(x+c)\right)=(1), \quad\left(f^{\prime \prime}(x), f^{\prime}(x+c) f^{\prime \prime}(x+c)\right)=(1)
$$

and hence $(f(x), f(x+c))=(1)$.

Let $\theta: F_{q} \rightarrow F_{q}$ be the Frobenius automorphism of $F_{q}$ over $F_{p}$, namely, $\theta(x)=x^{\prime \prime}$. Let $\chi$ be a multiplicative character of $F_{p}$. We denote by $\chi_{v}$ the character of $F_{q}$ induced by $\chi$ :

$$
\chi_{v}(x)=\chi\left(\operatorname{norm}_{v}(x)\right), \quad x \in F_{q},
$$

where

$$
\operatorname{norm}_{v}(x)=x \theta(x) \ldots \theta^{v-1}(x)=x x^{p} \ldots x^{p^{v-1}} .
$$

It is easy to see that if $p$ and $v$ are odd numbers, $\chi_{v}$ is induced by a non-trivial quadratic character of $F_{p}$, and $f(x)=\left(x+x^{(v)-1) 2}\right)\left(x+x^{p^{(v+1) / 2}}\right)$, then (see [1], Lemma 2)

$$
\chi_{v}(f(x))= \begin{cases}1 & \text { if } x \in F_{q}^{*} \\ 0 & \text { if } x=0\end{cases}
$$

Let

$$
\tilde{f}(x)=\frac{f(x)}{x^{2}}=\left(1+x^{p^{(v-1) / 2-1}}\right)\left(1+x^{p^{(v+1) 2-1}}\right) .
$$

Then $\chi_{v}(\tilde{f}(x))=1$ for all $x \in F_{q}$, and we have the following result. 
Lemma 3. Let $v>1$ be an odd integer, $F_{q}$ be a finite field of characteristic $p>2$ with $q=p^{v}$ elements, $c_{1}, \ldots, c_{s}$ be distinct elements of $F_{q}$, and let $N_{q}$ be the number of $F_{q}$-rational points of the affine curve $Y$ defined by the equations

$$
\begin{gathered}
z_{1}^{2}=f_{1}(x)=\tilde{f}\left(x+c_{1}\right), \\
z_{2}^{2}=f_{2}(x)=\tilde{f}\left(x+c_{2}\right), \\
\ldots \\
z_{s}^{2}=f_{s}(x)=\tilde{f}\left(x+c_{s}\right) .
\end{gathered}
$$

Then

$$
N_{q}=2^{s} q
$$

Proof. Since $\chi_{v}\left(f_{i}(x)\right)=\chi_{v}\left(\tilde{f}\left(x+c_{i}\right)\right)=1$ for all $x \in F_{p^{v}}, i=1, \ldots, s$, we have

$$
\begin{aligned}
N_{q} & =\sum_{x \in F_{p^{v}}}\left(1+\chi_{v}\left(f_{1}(x)\right)\right) \ldots\left(1+\chi_{v}\left(f_{s}(x)\right)\right) \\
& =\sum_{x \in F_{p^{v}}} 2^{s}=2^{s} p^{v}
\end{aligned}
$$

\section{PROOF OF THE THEOREM}

We consider the affine curve

$$
Y: \quad z_{i}^{2}=f_{i}(x)=\tilde{f}\left(x+c_{i}\right), \quad 1 \leq i \leq s,
$$

where $c_{1}, \ldots, c_{s}$ are distinct elements of $F_{q}$. The number of $F_{q}$-rational points of $Y$ is $N_{q}=2^{s} q$ by Lemma 3. The curve $Y$ satisfies the conditions of Lemma 1, so the genus $g=g(X)$ of its smooth projective model $X$ is

$$
g=2^{s-2}\left(\left(p^{(v-1) / 2}(p+1)-2\right) s-4\right)+1
$$

Let $S$ be the set of rational points on $Y$ and $S_{1} \subset S$ be a subset of $S$. Applying Goppa's construction to

$$
D_{0}=\sum_{P \in S_{1}} P
$$

and

$$
D=r P_{\infty},
$$

where $r<\operatorname{deg} D_{0}=\left|S_{1}\right|$ and $P_{\infty}$ is the point of $X$ corresponding to the point at infinity of the projectivization $\bar{Y}$ of the affine curve $Y$, we get $r<n \leq 2^{s} p^{v}, k \geq r+1-g, d \geq n-r$. Since in our case $2 g-2<r=\operatorname{deg} D<n$, we obtain $k=r+1-g$.

\section{REFERENCES}

1. S. A. Stepanov, Codes on fibre products of hyperelliptic curves. Discrete Math. Appl. (1997) 7, 77-88.

2. V. G. Goppa, Codes on algebraic curves, Soviet Math. Dokl. (1981) 24, 170-172.

3. I. R. Shafarevich, Basic Algebraic Geometry. Springer, Berlin, 1994. 